PSICOLOGÍA

IBEROAMERICANA
Psicología Iberoamericana ISSN: 1405-0943

revista.psicologia@ibero.mx

Universidad Iberoamericana, Ciudad de México México

\title{
El efecto de las percepciones de la enfermedad y de la utilidad de las medidas de prevención sobre la ejecución de conductas preventivas
}

Pérez-Bautista, Yuma Yoaly; Fernández-Vega, Margarita; Becerra-Gálvez, Ana Leticia; Reynoso-Erazo, Leonardo; Urzúa Morales, Alfonso; Lugo-González, Isaías Vicente

El efecto de las percepciones de la enfermedad y de la utilidad de las medidas de prevención sobre la ejecución de conductas preventivas

Psicología Iberoamericana, vol. 29, núm. 3, Esp., e293334, 2021

Universidad lberoamericana, Ciudad de México, México

Disponible en: https://www.redalyc.org/articulo.oa?id=133968747005

DOI: https://doi.org/10.48102/pi.v29i3.334

\section{(c) (1)}

Esta obra está bajo una Licencia Creative Commons Atribución 4.0 Internacional. 
Psicología Iberoamericana, vol. 29, núm. 3, Esp., e293334, 2021

Universidad Iberoamericana, Ciudad de México, México

Recepción: 01 Febrero 2021 Aprobación: 19 Abril 2021

DOI: https://doi.org/10.48102/ pi.v29i3.334

Redalyc: https://www.redalyc.org/ articulo.oa?id=133968747005
Artículos

\section{El efecto de las percepciones de la enfermedad y de la utilidad de las medidas de prevención sobre la ejecución de conductas preventivas}

The effect of illness perceptions and the usefulness of prevention measures on the execution of preventive

behaviours

Yuma Yoaly Pérez-Bautista yuma.yoaly@iztacala.unam.mx Universidad Nacional Autónoma de México, México

https://orcid.org/0000-0003-1032-1126 Margarita Fernández-Vega mfvg806@gmail.com Instituto Nacional de Enfermedades Respiratorias, México

https://orcid.org/0000-0001-6479-7889 Ana Leticia Becerra-Gálvez bega@comunidad.unam.mx Universidad Nacional Autónoma de México, México

(iD) https://orcid.org/0000-0002-5075-6098 Leonardo Reynoso-Erazo leoreynoso@gmail.com Universidad Nacional Autónoma de México, México D https://orcid.org/0000-0001-9793-1431 Alfonso Urzúa Morales alurzua@ucn.cl Universidad Católica del Norte, Chile https://orcid.org/0000-0002-0882-2194 Isaías Vicente Lugo-González isaiaslugo@iztacala.unam.mx Universidad Nacional Autónoma de México, México

https://orcid.org/0000-0002-2024-2598

Resumen: Desde el inicio de la pandemia por COVID-19, se hizo necesaria la implementación de estrategias para el mantenimiento de conductas para la prevención de contagios, sin embargo, no se ha logrado persuadir a toda la población de tomar dichas medidas. El uso de modelos psicológicos podría apoyar dando cuenta del proceso para la adopción de conductas que favorezcan la salud. Este estudio examina el rol mediador del control personal y la percepción de utilidad sobre las medidas de prevención en la relación entre percepción sobre la COVID-19 y la práctica de conductas de exposición durante la fase inicial de la pandemia. Se realizó un estudio cuantitativo transversalcorrelacional en el que se diseminó, por medio de correo electrónico y redes sociales, una batería de evaluación. A través de un muestreo en cadena participaron 370 mujeres y 370 hombres residentes de diferentes estados de la República Mexicana, quienes se encontraban entre los 18 y 73 años. Con el programa estadístico AMOS 25 se hizo un análisis de senderos, sobre el cual se reportan índices de ajuste absoluto y comparativo. El modelo mostró un buen ajuste, $\mathrm{X} 2=28.829, \mathrm{gl}=10, \mathrm{X} 2 / \mathrm{gl}=2.88, \mathrm{RMSEA}=.050$, $\mathrm{TLI}=.901$ y CFI $=.953$, corroborando que el control personal y la percepción de utilidad sobre las medidas de prevención favorecen la evitación de conductas de riesgo para infectarse de la COVID-19. 
Palabras clave: COVID-19, percepción de enfermedad, percepción sobre la conducta, Modelo de Sentido Común, conductas de exposición.

Abstract: Since the beginning of the COVID-19 pandemic, it was necessary to implement strategies to maintain behaviours for the prevention of infections, however, it has not been possible to persuade the entire population to take such measures. The use of psychological models helps account for the adoption of health-promoting behaviour. This study examines the mediating role of personal control and the perception of the usefulness of prevention measures in the relationship between perception of COVID-19 and the practice of exposure behaviours during the initial phase of the pandemic in a Mexican sample. A cross-correlational, quantitative study was carried out in which an evaluation battery was disseminated through email and social networks. Through chain sampling, 370 women and 370 men, who are residents of different states in Mexico, participated and were between the ages of 18 and 73 years old. With the statistical program AMOS 25, a path analysis was carried out, in which absolute and comparative adjustment indices were reported. The model showed a good fit, $\mathrm{X} 2=28.829, \mathrm{gl}=10$, $\mathrm{X} 2 / \mathrm{gl}=2.88, \mathrm{RMSEA}=.050, \mathrm{TLI}=.901$ and $\mathrm{CFI}=.953$, corroborating that personal control and the perception of utility on the measures of prevention favour the avoidance of risk behaviours to become infected with COVID-19.

Keywords: COVID-19, perception of illness, perceived behavioral, Common Sense Model, exposure behaviors..

\section{Introducción}

La pandemia provocada por la COVID-19, ha significado un desafío en las áreas sanitaria, educativa, social, económica y, por supuesto, la comportamental y la emocional (Douglas et al., 2020; Holmes et al., 2020; Lai et al., 2020; Mukhtar, 2020; Urzúa et al., 2020a). Desde el inicio de la pandemia, se ha documentado información sobre el impacto de ésta en diversas áreas, como por ejemplo en la salud mental y emocional en diversos sectores de la sociedad, donde se ha reportado la presencia de síntomas que van desde moderados a severos, en sintomatología relacionada con ansiedad, depresión, estrés, insomnio, solo por mencionar algunos (Alyami et al., 2020; Brooks et al., 2020; Lee, 2020; Mukhtar, 2020; Pérez-Fuentes et al., 2020; Pérez-Gay Juárez et al., 2020; Samaniego et al., 2020; Sandín et al., 2020; Urzúa et al., 2020a; Wang et al., 2020).

Las instituciones responsables del manejo de la pandemia se han encontrado, por un lado, con diversas dificultades para su afrontamiento, entre ellas las relacionadas con la adquisición y el mantenimiento de comportamientos para prevenir el contagio por SARS-CoV-2. Por otro, el abandono de comportamientos seguros, aumentando la probabilidad de contagio, entre los que destacan las medidas conductuales como el distanciamiento social (permanecer en casa, solo salir para actividades esenciales), la higiene individual (lavado de manos con agua y jabón o gel antibacterial) y la evitación de conductas de riesgo social (evitar las aglomeraciones, contacto físico, saludo de mano y saludo de beso). Estas últimas fueron determinadas como las principales medidas a seguir desde el inicio de la pandemia (Berg \& Lin, 2020; Chater et al., 2020; Gallegos et al., 2020; Hagger et al., 2020; Holmes et al., 2020; Lin et al., 2020; Secretaría de Salud [SSa], 2020; West et al., 2020).

La evidencia actual y la aportada por las investigaciones realizadas durante las epidemias de Influenza H1N1 en 2009 y del virus MERS en 
2014, basados en modelos clásicos de cambio conductual en salud, tales como el Modelo de Creencias de Salud (Rosenstock, 1974), Teoría de la Conducta Planeada (Ajzen \& Fishbein, 1980) y Teoría de la Acción Razonada (Ajzen, 1991), han mostrado que existen factores psicosociales que predicen conductas de prevención y la evitación de conductas de riesgo. Entre estos factores destacan aquellos centrados en la percepción sobre la enfermedad (percepción de la amenaza, susceptibilidad percibida, severidad percibida) y los centrados en las conductas (autoeficacia, locus de control, actitudes hacia la conducta, percepción de otros sobre el comportamiento y las intenciones de realizar la conducta (Berg \& Lin, 2020; Hagger et al., 2020; Lin et al., 2020; Rubin et al., 2010; Taylor, 2019; Urzúa et al., 2020b; West et al., 2020; Yang \& Cho, 2017). Estos hallazgos han aportado evidencia a la hipótesis de que las personas se comportarán de manera preventiva o no, en función de cómo perciban la enfermedad que podrían contraer (percepción de riesgo, percepción de gravedad y amenaza), y/o de cómo perciban las conductas mediante las cuales podrían evitar contraer dicha enfermedad (autoeficacia, percepción de utilidad personal y del grupo de referencia e intenciones de realizar las conductas).

Uno de los modelos que toma como base la percepción de amenaza, y que podría explicar la implementación de conductas de protección a la salud, es el Modelo de Sentido Común y Percepción de la Enfermedad (MSCPE), propuesto por Leventhal et al. (1980). Según éste, un individuo podría ejecutar comportamientos que protejan su salud dependiendo de cómo percibe una enfermedad o una amenaza a su salud. Esta percepción sería de tipo cognitiva y emocional, subdividiéndose en: identidad, temporalidad, causas, consecuencias, control personal, coherencia . percepción emocional (Broadbent et al., 2006; Leventhal et al., 1980; Moss-Morris et al., 2002). Diversos autores han sugerido que este modelo podría obtener mejores resultados explicativos y predictivos sobre comportamiento de protección para la salud si se incluyeran variables relativas a la percepción sobre las conductas de protección a la salud, la percepción de los tratamientos para una enfermedad o la capacidad para realizar dichos comportamientos (Aujla et al., 2016; Brandes \& Mullan, 2014; Breland et al., 2020; Hagger et al., 2017; Hagger \& Orbell, 2021; Holmes et al., 2014; Horne et al., 2013; Kucukarslan, 2012; Unni \& Shiyanbola, 2016).

En la presente investigación, y dado el contexto pandémico, se ha considerado la percepción que tienen los participantes sobre la enfermedad, las medidas de prevención y el efecto que tiene ésta sobre la ejecución de comportamientos vinculados a la prevención del contagio del coronavirus causante de la COVID-19, por ejemplo, el distanciamiento físico (quedarse o evitar salir de casa, evitar el contacto con otras personas) y medidas de higiene y seguridad (lavado de manos en caso de contacto, uso de mascarillas). El supuesto de esta relación se desprende de propuestas teóricas, desde las que se determina una diferencia entre la percepción sobre la enfermedad (objeto) y la percepción sobre la conducta, existiendo una relación más cercana entre 
estas últimas y las conductas de prevención, dado el aspecto conductual de ambas (Ajzen \& Fishbein, 1980).

Considerando las sugerencias hechas al modelo tradicional del MSCPE, hemos incorporado dos variables que pudiesen tener un efecto mediador, las percepciones de control de la enfermedad y de la utilidad de las medidas preventivas. En este contexto, se ha documentado que, una elevada percepción de control sobre la enfermedad y de la utilidad de los tratamientos, una comprensión clara de la enfermedad (coherencia), así como una elevada percepción de consecuencias negativas derivadas de la enfermedad, se relaciona con la práctica de comportamientos de protección a la salud, aunque esto se ha documentado en pacientes con enfermedades crónicas (Aujla et al., 2016; Brandes \& Mullan, 2014; Hagger \& Orbell, 2003; Kaptein et al., 2008, 2010; Kucukarslan, 2012; Law et al., 2014).

Algunas investigaciones similares en el contexto de la COVID-19 han propuesto modelos explicativos a través de Modelamiento de Ecuaciones Estructurales (SEM por sus siglas en inglés) para conductas de distanciamiento social y conductas de prevención en personas de Norteamérica, Europa y Asia, aunque partiendo de la integración de los modelos clásicos de cambio conductual mencionados previamente. También, han propuesto la inclusión de variables mediadoras como la percepción de riesgo, la susceptibilidad percibida, la autoeficacia y la percepción sobre la efectividad de las medidas de prevención (Anaki \& Sergay, 2021; Hagger et al., 2020; Hamilton et al., 2020; IrigoyenCamacho et al., 2020; Lin et al., 2020; Raude et al., 2020; Shahnazi et al., 2020).

Tomando como base lo anterior, este estudio tuvo el propósito de examinar el rol mediador del control personal y la percepción de utilidad sobre las medidas de prevención en la relación entre percepción sobre la COVID-19 y la práctica de conductas de exposición durante la fase inicial de la pandemia en México.

\section{Método}

\section{Diseño}

De acuerdo con Hernández-Sampieri et al. (2014) se realizó un estudio cuantitativo, mediante un diseño no experimental, transversal y correlacional.

\section{Participantes}

A través de un muestreo no representativo, de tipo cadena o por redes (Hernández-Sampieri et al., 2014) participaron, de manera voluntaria, 740 adultos de diferentes estados de la República Mexicana. En la Tabla 1 se describen las características de los participantes. 
Tabla 1

Tabla 1

Características sociodemográficas de los participantes

\begin{tabular}{|lcc|}
\hline \multicolumn{1}{c}{ Variables } & $n$ & $\%$ \\
\hline Sexo & & \\
\hline Hombre & 370 & 50 \\
\hline Mujer & 370 & 50 \\
\hline Residencia & 280 & 39.1 \\
\hline Ciudad de México & 257 & 34.7 \\
\hline Estado de México & 194 & 25.2 \\
\hline Interior de la República & & \\
\hline Estado civil & 415 & 56.1 \\
\hline Solteros & 177 & 23.9 \\
\hline Casados & 109 & 14.7 \\
\hline Unión libre & 39 & 5.3 \\
\hline Otro & & \\
\hline Nivel educativo & 526 & 71.1 \\
\hline Licenciatura & 88 & 11.9 \\
\hline Preparatoria & 77 & 10.4 \\
\hline Posgrado & 31 & 4.2 \\
\hline Técnico & 18 & 2.4 \\
\hline Básico & & \\
\hline Actividad laboral & 264 & 35.7 \\
\hline Profesionista & 178 & 24.1 \\
\hline Empleado & 163 & 22 \\
\hline Estudiante & 135 & 18.2 \\
\hline Otro & & \\
\hline & & \\
\hline & $M_{\text {endad }}=32.82$ años $(D E=10.789)$, Rango $=18-73$ años \\
\hline
\end{tabular}

\section{Instrumentos}

\section{Cédula de datos sociodemográficos}

Conjunto de ítems para reunir información sobre datos de residencia, familiares, educativos, ocupacionales, entre otros.

Cuestionario Revisado de Percepción de Enfermedad-COVID19 (IPQ-RCOVID19)

Instrumento diseñado para evaluar la percepción cognitiva y emocional de la COVID-19 en México (Lugo-González et al., 2020). De este instrumento se utilizaron en el presente estudio las dimensiones de a) Consecuencias, compuesta por cinco ítems, la cual evalúa las repercusiones que el sujeto percibe que podría tener la enfermedad en su vida. La confiabilidad de esta dimensión fue de $\Omega=.86$, b) Control personal, compuesta por tres ítems, la cual inquiere sobre la percepción del 
grado de control que el sujeto percibe que tiene sobre prevenir infectarse, con una confiabilidad de $\Omega=.78$ y c) Percepción emocional, la cual a través de seis ítems evalúa la repercusión emocional de la enfermedad en el sujeto. Esta dimensión tiene una confiabilidad de $\Omega=.94$. Toda la escala cuenta con un formato de respuesta tipo Likert de cuatro puntos que va de totalmente en desacuerdo, a totalmente de acuerdo.

\section{Evaluación de Percepción sobre las Medidas de Prevención-COVID-19 (EPMP-COVID19)}

Ítems diseñados ad hoc para el presente estudio, la cual está constituida por seis ítems que evalúan la percepción sobre la utilidad de las medidas para prevenir el contagio de la COVID-19 basadas en el comportamiento de distanciamiento social (quedarse o evitar salir de casa, evitar el contacto con otras personas y recurrir al lavado de manos en caso de contacto). La evaluación cuenta con un formato de respuesta tipo Likert de cuatro puntos que va de totalmente en desacuerdo, a totalmente de acuerdo. El coeficiente omega $(\Omega)$ fue de .95

\section{Escala de Conductas de Exposición COVID-19 (ECE-COVID19 Lugo-} González et al., 2020)

Escala conductual constituida por cinco ítems que evalúan la frecuencia de conductas de riesgo (contacto físico, saludo de mano y saludo de beso, estar cerca de personas y acudir a lugares). La escala cuenta con un formato de respuesta tipo Likert de cuatro puntos que va de siempre lo hago así, a nunca lo hago así. Para este estudio el análisis de confiabilidad mostró un coeficiente omega $(\Omega)$ de .86

\section{Procedimiento}

La batería de evaluación se desarrolló en la plataforma Google-Forms Online $\mathrm{y}$ fue diseminada por medio de correo electrónico y redes sociales como Facebook $^{\circ}$ y WhatsApp entre el 22 de marzo y el cuatro de abril de 2020 .

\section{Aspectos éticos}

Los participantes podían responder el formulario con previa aceptación de la información bajo su consentimiento. El proyecto fue evaluado y aceptado por el comité de ética en investigación del Instituto Nacional de Enfermedades Respiratorias (INER), bajo el siguiente número de registro asignado por el comité: S02-20. 


\section{Análisis de resultados}

Los datos se analizaron con el programa estadístico SPSS versión 24 y AMOS 25 para Windows. Se emplearon estadísticos descriptivos para resumir las características de los participantes y las variables de estudio. La normalidad de la distribución de los datos se evaluó de acuerdo con los coeficientes de asimetría (valor) <1.5) y curtosis (valor) <1.5), y la razón crítica de curtosis multivariante (valor) <7.00) (Hong et al., 2020).

Primero, se estimaron los modelos de medida de cada escala a través del Análisis Factorial Confirmatorio (AFC), tomando como criterio los índices de ajuste mediante la ponderación de regresión estandarizada (cargas factoriales) y los siguientes índices de ajuste: el estadístico chicuadrado $\left(\chi^{2}, p>.05\right)$, el cociente resultante de $\chi^{2} / g l,(<3)$, el Error cuadrático medio de aproximación (RMSEA $<.08,90 \%$ IC), el Índice de ajuste comparativo (CFI > .90) y el Índice de Tucker-Lewis (TLI > .90; Pérez et al., 2013); como se describe en la Tabla 3

La confiabilidad interna de las mediciones se examinó utilizando el coeficiente omega $(\Omega)$.

Posteriormente, se realizó un análisis preliminar de correlación $R$ ho de Spearman con el propósito de derivar hipótesis con base en los supuestos del MSCPE para la posterior construcción de un modelo de mediación múltiple. Dicho modelo hipotético se probó con análisis SEM, utilizando la estimación de máxima verosimilitud con Bootstrapping y Bias corregido del 95\% (BC) con 5000 muestras (Lin et al., 2020; Pérez et al., 2013), además de considerar los índices y criterios ya mencionados en la evaluación del modelo de medida.

En el modelo SEM se buscó probar si la percepción negativa sobre la COVID-19 (variable latente construida con percepción emocional y consecuencias percibidas) generaba un efecto positivo sobre el control personal y la percepción de utilidad sobre medidas preventivas, empero no un efecto directo sobre las conductas de exposición, además de evidenciar la relación directa entre el control personal y la percepción de utilidad sobre medidas preventivas, la cual podría estar explicando el comportamiento de exposición (no prevención) durante la primera etapa de la pandemia. Finalmente, se calcularon las estimaciones estándar de los efectos directos, indirectos y totales para determinar la asociación entre las variables latentes y observadas.

\section{Resultados}

En la Tabla 2 se pueden observar las relaciones entre cada una de las variables analizadas, para los propósitos del estudio, las de menor relevancia son las que pertenecen a subdimensiones del IPQ-RCOVID19 y el ECE-COVID19, siendo estas, mínimas $(R h o=-104$ a $-.079, p=<.01)$. Por el contrario, y con una mayor fuerza se ubican las del IPQ-R-COVID19 y EPMP-COVID19 $(R h o=.267$ a $.377, p=$ 
$<.01)$, seguida del EPMP-COVID19 y el ECE-COVID19 $(R h o=-.158$, $p=<.01)$.

Estos datos podrían sugerir que, la percepción sobre la utilidad de las medidas de prevención y el control personal podrían mediar la relación entre la percepción de la COVID-19 y las conductas de exposición, al inicio de la pandemia.

Tabla 2

Tabla 2

\begin{tabular}{|c|c|c|c|c|c|}
\hline Instrumentos & Variables & 1 & 2 & 3 & 4 \\
\hline IPQ-R-COVID19 & $\begin{array}{l}\text { 1. Percepción Emocional } \\
\text { 2. Consecuencias percibidas } \\
\text { 3. Control personal }\end{array}$ & $\begin{array}{l}- \\
.451 * * \\
.132 * *\end{array}$ & $304 * *$ & - & \\
\hline EPMP-COVID19 & $\begin{array}{l}\text { 4. Percepción de utilidad de } \\
\text { medidas }\end{array}$ & $.267 * *$ & $.320 * *$ & $.377 * *$ & - \\
\hline ECE-COVID19 & 5. Conductas de exposición & $-.086 * *$ & $-.079 *$ & $-.104^{* * * *}$ & $-.158 * *$ \\
\hline
\end{tabular}

Nota: $* p=<.05 ; * * p=<.01 ; * * * p=<.01$

\section{Análisis de mediación múltiple}

Se analizó un modelo de mediación múltiple de la percepción de utilidad sobre las medidas de prevención y el control personal (capacidad percibida para prevenir infectarse de la COVID-19) sobre la relación entre la percepción sobre la COVID-19 (impacto emocional y consecuencias psicosociales) y las conductas de exposición (Figura 1).

Figura 1

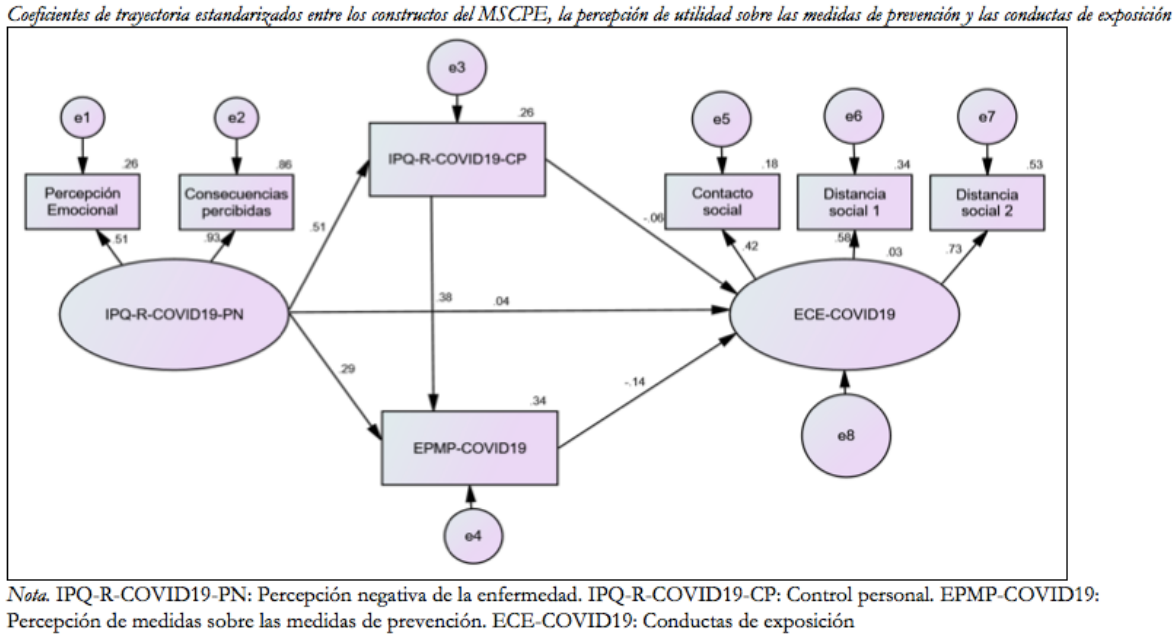

Figura 1

Este modelo presenta indicadores que pueden ser considerados como de un buen ajuste (Ver Tabla 3), mostrando que los efectos más importantes se observan entre las variables perceptuales (percepción de enfermedad y de la utilidad sobre las medidas de prevención), no así con el auto reporte de conductas de exposición. 
Tabla 3

Tabla 3

\begin{tabular}{|c|c|c|c|c|c|c|c|c|c|}
\hline \multirow{2}{*}{ Modelos } & \multirow{2}{*}{ Parámetro } & \multirow{2}{*}{$X^{2}$} & \multirow{2}{*}{ DF } & \multirow{2}{*}{$p$} & \multirow{2}{*}{ CFI } & \multirow{2}{*}{ TLI } & \multirow{2}{*}{ RMSEA } & \multicolumn{2}{|c|}{ RMSEA IC $90 \%$} \\
\hline & & & & & & & & Bajo & Alto \\
\hline $\begin{array}{l}\text { IPQ-R- } \\
\text { COVID19 }\end{array}$ & 31 & 246.007 & 74 & .000 & .962 & .954 & .056 & .048 & .064 \\
\hline $\begin{array}{l}\text { EPMP- } \\
\text { COVID19 }\end{array}$ & 11 & 2.864 & 4 & .581 & 1.000 & 1.003 & .000 & .000 & .048 \\
\hline EPE-COVID19 & 13 & 14.734 & 8 & .065 & .999 & .998 & .034 & .000 & .060 \\
\hline Modelo & 18 & 28.829 & 10 & .001 & .953 & .901 & .050 & .030 & .073 \\
\hline
\end{tabular}

A pesar de esto, se identifica que los efectos entre la percepción negativa sobre la COVID-19 y las conductas de exposición son positivos, aunque muy débiles (efectos directos $=.042$, efectos indirectos $=-.102 \mathrm{y}$ efectos totales $=-.060)$, los cuales, mejoran al incluir en la relación al control personal (efectos directos $=-.064$. efectos indirectos $=-.054 \mathrm{y}$ efectos totales $=-.118)$, y la percepción de utilidad sobre las medidas de prevención (efectos directos y efectos totales $=-.144 \mathrm{e}$ indirectos $=-.000$ ), además de ser negativos. Esto implica que una percepción negativa sobre la COVID-19, sentirse capaz de prevenir infectarse de SARS CoV -2 y considerar que las medidas de prevención son útiles, podría derivar en que una persona no ponga en práctica conductas de riesgo para contraer la enfermedad

\section{Discusión}

El presente estudio tuvo como propósito evaluar el rol mediador del control personal y la percepción de utilidad sobre las medidas de prevención en la relación entre percepción sobre la COVID-19 y la práctica de conductas de exposición (como no seguir las medidas de distanciamiento social y lavado de manos) durante la fase inicial de la pandemia, con base en la estrategia inicial en pro de prevenir contagios (Güner et al., 2020), controlar la saturación hospitalaria y garantizar la atención médica (SSa, 2020).

Este estudio permite comprender cómo es que factores psicológicos se involucran en la evitación de conductas de exposición al virus y en consecuencia enfermar de la COVID-19. Los resultados muestran que evitar comportamientos de riesgo estaría en función de las experiencias perceptuales en términos emocionales (percepción emocional sobre la COVID-19) y consecuencias futuras de la enfermedad, así como por la capacidad percibida para prevenir contagiarse (control personal) y de una percepción positiva sobre la utilidad de las medidas preventivas. Resultados similares se reportan en el estudio de Li et al. (2020), donde se identificó que percibir el virus como grave se relaciona con un impacto 
negativo a nivel emocional por el brote de COVID-19, en contraste, percibir que el virus es controlable se relaciona con un mejor ajuste emocional. Esto también se observa en los estudios de Hamilton et al. (2020), Hagger et al. (2020) y Lin et al. (2020) donde se evidencia la importancia de las variables vinculadas con el control personal (control conductual percibido, autoeficacia e intención conductual) sobre las conductas de prevención y evitación de comportamientos de exposición.

No obstante, es importante tomar en cuenta que la relación entre el control personal y la percepción de utilidad sobre las medidas de prevención fueron débiles. Una posible explicación que justifica estos resultados es el momento en el que este estudio se llevó a cabo, debido a que una de las primeras medidas para el control de la epidemia fue el cierre de la mayoría de las actividades económicas, educativas y recreativas que favorecían la interacción social (SSa, 2020). Por lo tanto, la evitación de conductas de exposición obedecía, en mayor medida, a las restricciones para el contacto social, no así, a la propia disposición de las personas para evitar dichos comportamientos (Güner et al., 2020).

Aunque el aislamiento social ha sido una de las medidas más controversiales, se dispone de evidencia sobre su eficacia para mitigar los efectos de la pandemia (Güner et al., 2020) sobre todo en la etapa inicial de la enfermedad. Esto, debido a que se sugiere que las personas que inician en la enfermedad pueden infectar a otras inadvertidamente, antes de darse cuenta de que se encuentran infectadas porque aún no han experimentado síntomas (Hartley et al., 2020), de ahí que sea un comportamiento que debe seguir analizándose.

Al mismo tiempo, los resultados obtenidos en el presente estudio deben de ser leídos en el contexto socioeconómico del país, ya que, si bien las medidas de distanciamiento social fueron adoptadas en países de Asia, Europa y Norteamérica, pudieran ser menos efectiva en aquellos que cuentan con un alto índice poblacional y que viven en pobreza extrema (Barnett-Howell \& Mushfiq, 2020). Esto, debido a que, las personas que viven en un contexto económico hostil son los menos dispuestos a llevar a cabo comportamientos que comprometan su economía, pues el valor que le otorgan a las consecuencias económicas está por encima de aquellas derivadas por la enfermedad (Barnett-Howell \& Mushfiq, 2020).

Además, es importante considerar que la intervención de otras variables en el modelo podría mejorar sus efectos explicativos, por ejemplo: percepción de riesgo, severidad atribuida a la COVID-19, impacto emocional, formas de afrontamiento (Guzmán-González et al., 2020; Irigoyen-Camacho et al., 2020; Lugo-González et al., 2020; RamosLira et al., 2020), confianza en las autoridades de salud, evolución de la pandemia o el rol de los medios de comunicación y las redes sociales (Garfin et al., 2020; Liu \& Mesch, 2020; Lugo-González et al., 2021; Lunn et al., 2020; Rubin et al., 2010).

Las principales limitaciones del estudio se vincularían, con la evaluación por medios digitales y diseminación del instrumento, ya que se pueden considerar inexactos o métodos donde se minimiza o sobreestima el autoinforme de las variables evaluadas, además de que pueden tener un 
sesgo durante el muestreo, a pesar de ello, también hay evidencias sobre su utilidad, funcionalidad y equivalencia con las formas de evaluación tradicional (Pérez-Bautista \& Lugo-González, 2017; Stirratt et al., 2015; Weigold et al., 2013).

Finalmente, con este trabajo se busca contribuir a la pandemia causada por la enfermedad COVID-19, resaltado la importancia del análisis del comportamiento de exposición y las variables psicológicas asociadas, pues a pesar de que ha transcurrido más de un año desde que se detectó el primer caso en México (27 de febrero de 2020), la reducción de contagios ha sido una labor complicada y en medida que han transcurrido los meses el distanciamiento social autoimpuesto ha disminuido. En consecuencia, se han implementado otras medidas como el uso del cubrebocas, que, si bien han demostrado muy buena efectividad para la prevención de contagios (Manikandan, 2020), son menos efectivas en comparación con el distanciamiento social autoimpuesto y más aún con el distanciamiento social impuesto (Manikandan, 2020; Teslya et al., 2020).

\section{Conclusiones}

Esa investigación brinda evidencia sobre el impacto de las variables perceptuales, emocionales y de control personal sobre la evitación de comportamientos que pongan en riesgo la salud de las personas ante la COVID-19, asimismo se enfatiza la importancia de dar continuidad a este tipo de investigaciones, dada la diseminación variada virus SARS-CoV-2 y su impacto en el contexto social. También se considera importancia del trabajo psicológico en el terreno de la salud, pues el análisis de las variables psicológicas permiten hacer propuestas basadas en evidencia para la promoción y mantenimiento de conductas protectoras para el regreso a la nueva normalidad. A pesar de que se ha dado inicio al programa nacional de vacunación, éste será un proceso de larga duración y en el que se sigue solicitando la adhesión de comportamientos de prevención y la evitación de comportamientos de riesgo en el actuar diario, independientemente se considera vacunarse o está por hacerlo.

\section{Referencias}

Ajzen, I. (1991). The theory of planned behavior. Organizational Behavior and Human Decision Process, 50, 179-211. https://doi.org/10.1016/0749-59 78(91)90020-T

Ajzen, I., \& Fishbein, M. (1980). Understanding attitudes and predicting social behavior. Prentice-Hall.

Alyami, M., Henning, M., Krägeloh, C. U., \& Alyami, H. (2020). Psychometric evaluation of the Arabic version of the fear of COVID-19 scale. International Journal of Mental Health and Addiction, 1. https://doi.org/ 10.1007/s11469-020-00316-x

Anaki, D., \& Sergay, J. (2021). Predicting health behavior in response to the coronavirus disease (COVID-19): Worldwide survey results from early 
March 2020. Plosone,16(1). https://doi.org/10.1371/journal.pone.0244 534

Aujla, N., Walker, M., Sprigg, N., Abrams, K., Massey, A., \& Vedhara, K. (2016). Can illness beliefs, from the common-sense model, prospectively predict adherence to self-management behaviours? A systematic review and metaanalysis. Psychology \& Health, 31 (8). 931-958. https://doi.org/10.1080/ 08870446.2016.1153640

Barnett-Howell, Z., \& Mobarak, A. M. (2020). The benefits and costs of social distancing in rich and poor countries. arXiv. https://arxiv.org/pdf/2004 .04867.pdf

Berg, M. B., \& Lin, L. (2020). Prevalence and predictors of early COVID-19 behavioral intentions in the United States. Translational Behavioral Medicine, 10(4), 843-849. https://doi.org/10.1093/tbm/ibaa085

Brandes, K., \& Mullan, B. (2014). Can the common-sense model predict adherence in chronically ill patients? A meta-analysis. Health Psychology Review, 8(2), 129-153. https://doi.org/10.1080/17437199.2013.820986

Breland, J. Y., Wong, J. J., \& McAndrew, L. M. (2020). Are Common Sense Model constructs and self-efficacy simultaneously correlated with selfmanagement behaviors and health outcomes: A systematic review. Health Psychology Open, 7(1), https://doi.org/10.1177/2055102919898846

Broadbent, E., Petrie, K., Main, J., \& Weinman, J. (2006). The brief illness perception questionnaire. Journal of Psychosomatic Research. 60, 631-637. https://doi.org/10.1016/j.jpsychores.2005.10.020

Brooks, S. K., Webster, R. K., Smith, L. E., Woodland, L., Wessely, S., Greenberg, N., \& Rubin, G. J. (2020). The psychological impact of quarantine and how to reduce it: Rapid review of the evidence. The Lancet, 395(10227), 912-920. http://doi.org/10.1016/S0140-6736(20)304608

Chater, A. M., Arden, M., Armitage, C., Byrne-Davis, L., Chadwick, P., Drury, J., Hart, J., Lewis, L., McBride, E., Perriard-Abdoh, S., Thompson, S., Whittaker, E., \& O'Connor, D. (2020). Behaviouralscience and disease prevention: Psychological guidance. British Psychological Society. https:// www.bps.org.uk/

Douglas, M., Katikireddi, S. V., Taulbut, M., McKee, M., \& McCartney, G. (2020). Mitigating the wider health effects of covid-19 pandemic response. BMJ, 369, 1-6. https://doi.org/10.1136/bmj.m1557

Gallegos, M., Zalaquett, C., Luna, S., Mazo-Zea, R., Ortiz-Torres, B., PenagosCorso, J., Portillo, N., Torres, I., Urzúa, A., Morgan, M., Polanco, F., Florez, A., \& Lopes, R. (2020). Cómo afrontar la pandemia del coronavirus (covid-19) en las américas: recomendaciones y líneas de acción sobre salud mental. Revista Interamericana de Psicologia, 54(1), https://d oi.org/10.30849/ripijp.v54i1.1304

Garfin, D. R., Silver, R. C., \& Holman, E. A. (2020). The novel coronavirus (COVID-2019) outbreak: Amplification of public health consequences by media exposure. Health Psychology. http://dx.doi.org/10.1037/hea00 00875

Guzmán-González, J. I., Sánchez-García, F. G., Ramírez-de los Santos, S., Gutiérrez-Rodríguez, F., Palomino-Esparza, D., \& Telles-Martínez, A. L. (2020). Worry and perceived risk of contagion during the COVID-19 
quarantine in the Jalisco population: Preliminary study. Salud Mental, 43(6), 253-261. https://doi.org/10.17711/SM.0185-3325.2020.035

Güner, R., Hasanoğlu, I., \& Aktaş, F. (2020). COVID-19: Prevention and control measures in community. Turkish Journal of Medical Sciences, 50(SI-1), 571-577. https://doi.org/10.3906/sag-2004-146

Hagger, M. S., Koch, S., Chatzisarantis, N. L., \& Orbell, S. (2017). The common sense model of self-regulation: Meta-analysis and test of a process model. Psychological Bulletin, 143(11), 1117-1154. https://doi.org/10.1037/bul 0000118

Hagger, M. S., \& Orbell, S. (2003). A meta-analytic review of the common-sense model of illness representations. Psychology and Health, 18(2), 141-184. h ttps://doi.org/10.1080/088704403100081321

Hagger, M. S., \& Orbell, S. (2021). The common sense model of illness selfregulation: A conceptual review and proposed extended model. Health Psychology Review, 1-57. https://doi.org/10.1080/17437199.2021.18780 50

Hagger, M. S., Smith, S. R., Keech, J. J., Moyers, S. A., \& Hamilton, K. (2020). Predicting social distancing intention and behavior during the COVID-19 pandemic: An integrated social cognition model. Annals of Behavioral Medicine, 54(10), 713-727. https://doi.org/10.1093/abm/ka aa073

Hamilton, K., Smith, S. R., Keech, J. J., Moyers, S. A., \& Hagger, M. S. (2020). Application of the Health Action Process Approach to social distancing behavior during COVID-19. Applied Psychology: Health and Well\#Being. https://doi.org/10.1111/aphw.12231

Hartley, D. M., Reisinger, H. S., \& Perencevich, E. N. (2020). When infection prevention enters the temple: Intergenerational social distancing and Covid-19. Infection Control \& Hospital Epidemiology, 41(7), 868-869. ht tps://doi.org/10.1017/ice.2020.100

Hernández-Sampieri, R., Fernández-Collado, C., \& Baptista-Lucio, P. (2014). Metodología de la investigación. Mc Graw-Hill.

Holmes, E. A., Hughes, D. A., \& Morrison, V. L. (2014). Predicting adherence to medications using health psychology theories: A systematic review of 20 years of empirical research. Value in Health, 17(8), 863-876. https://d oi.org/10.1016/j.jval.2014.08.2671

Holmes, E. A., O'Connor, R. C., Perry, V. H., Tracey, I., Wessely, S., Arseneault, L., Ballard, C., Christensen, C., Cohen-Silver, R., Everall, I., Ford, T., John, A., Kabir, T., King, K., Madan, I., Michie, S., Przybylski, P., Shafran, R., Sweeney, A., Worthman, C., Yardley, L., Cowan, K., Cope, C., Hotopf, M., \& Bullmore, E. (2020). Multidisciplinary research priorities for the COVID-19 pandemic: A call for action for mental health science. The Lancet Psychiatry. 1-14. https://doi.org/10.1016/S2215-0366(20)30168 $-1$

Hong, S. J., Shin, N. M., \& Jung, S. (2020). A predictive model of fear of cancer recurrence for patients undergoing chemotherapy. Supportive Care in Cancer, 28(9), 4173-4181. https://doi.org/10.1007/s00520-019-0524 5-7

Horne, R., Chapman, S. C., Parham, R., Freemantle, N., Forbes, A., \& Cooper, V. (2013). Understanding patients' adherence-related beliefs about medicines prescribed for long-term conditions: A meta-analytic 
review of the Necessity-Concerns Framework. PloS one, 8(12), 1-24. http s://doi.org/10.1371/journal.pone.0080633

Irigoyen-Camacho, M. E., Velazquez-Alva, M. C., Zepeda-Zepeda, M. A., Cabrer-Rosales, M. F., Lazarevich, I., \& Castaño-Seiquer, A. (2020). Effect of income level and perception of susceptibility and severity of COVID-19 on stay-at-home preventive behavior in a group of older adults in Mexico City. International Journal of Environmental Research and Public Health, 17(20), 7418. https://doi.org/10.3390/ijerph 17207418

Kaptein, A. A., Hughes, B. M., Scharloo, M., Fischer, M. J., Snoei, L., Weinman, J., \& Rabe, K. F. (2008). Illness perceptions about asthma are determinants of outcome. Journal of Asthma, 45(6), 459-464. https://doi.org/10.1080 /02770900802040043

Kaptein, A. A., Klok, T., Moss-Morris, R., \& Brand, P. L. (2010). Illness perceptions: impact on self-management and control in asthma. Current Opinion in Allergy and Clinical Immunology, 10(3), 194-199. https://doi .org/10.1097/ACI.0b013e32833950c1

Kucukarslan, S. N. (2012). A review of published studies of patients' illness perceptions and medication adherence: Lessons learned and future directions. Research in Social and Administrative Pharmacy, 8(5), 371-382. https://doi.org/10.1016/j.sapharm.2011.09.002

Lai, J., Ma, S., Wang, Y., Cai, Z., Hu, J., Wei, N., Wu, J., Du, H., Chen, T., Li, R., Tan, H., Kang, H., Yao, L., Huang, M., Wang, H., Wang, G., Liu, Z., $\& \mathrm{Hu}, \mathrm{Z}$. (2020). Factors associated with mental health outcomes among health care workers exposed to coronavirus disease 2019. JAMA Network Open, 3(3), e204006-12. http://doi.org/10.1001/jamanetworkopen.202 0.3976

Law, G. U., Tolgyesi, C. S., \& Howard, R. A. (2014). Illness beliefs and self-management in children and young people with chronic illness: A systematic review. Health Psychology Review, 8(3), 362-380. https://doi.o $\mathrm{rg} / 10.1080 / 17437199.2012 .747123$

Lee, S. A. (2020). Measuring coronaphobia: The psychological basis of the Coronavirus Anxiety Scale. Neurological Sciences, 33, 0-0. https://doi.org /10.14744/DAJPNS.2020.00069

Leventhal, H., Meyer, D., \& Nerenz, D. (1980). The common sense representation of illness danger. En S. Rachman (Ed.), Contributions to medical psychology volume II (pp. 9-33). Pergamon Press.

Li, J. B., Yang, A., Dou, K., Wang, L. X., Zhang, M. C., \& Lin, X. Q. (2020). Chinese public's knowledge, perceived severity, and perceived controllability of COVID-19 and their associations with emotional and behavioural reactions, social participation, and precautionary behaviour: A national survey. BMC Public Health, 20(1), 1-14. https://doi.org/10.1 186/s12889-020-09695-1

Lin, C. Y., Imani, V., Majd, N. R., Ghasemi, Z., Griffiths, M. D., Hamilton, K., Hagger, M. S., \& Pakpour, A. H. (2020). Using an integrated social cognition model to predict COVID - 19 preventive behaviours. British Journal of Health Psychology, 25(4), 981-1005. https://doi.org/10.1111/ bjhp. 12465

Liu, X. J., \& Mesch, G. S. (2020). The adoption of preventive behaviors during the COVID-19 pandemic in China and Israel. International Journal of 
Environmental Research and Public Health, 17(19), https://doi.org/10.3 390/ijerph 17197170

Lugo-González, I. V., Fernández-Vega, M., Reynoso-Erazo, L., Becerra-Gálvez, A. L., \& Pérez-Bautista, Y. Y. (2020). COVID-19 perception and preventive behaviors: A descriptive, comparative study by severity and perceived risk. Salud Mental, 43(6), 285-292. https://doi.org/10.17711/ SM.0185-3325.2020.039

Lugo-González, I., Pérez-Bautista, Y., Becerra-Gálvez, A., Fernández-Vega, M., \& Reynoso-Erazo, L. (2021). Percepción emocional del COVID-19 en México: estudio comparación entre la fase 1 , fase 2 y medios de información. Interacciones, 7, e164. https://doi.org/10.24016/2021.v7.1 64

Lunn, P. D., Belton, C. A., Lavin, C., McGowan, F. P., Timmons, S., \& Robertson, D. A. (2020). Using behavioral science to help fight the Coronavirus. Journal of Behavioral Public Administration, 3(1). https://d oi.org/10.30636/jbpa.31.147

Manikandan, N. (2020). Are social distancing, hand washing and wearing masks appropriate measures to mitigate transmission of COVID-19? Vacunas, 21(2), 136. https://doi.org/10.1016/j.vacun.2020.09.001

Moss-Morris, R., Weinman, J., Petrie, K., Horne, R., Cameron, L., \& Buick, D. (2002). The Revised Illness Perception Questionnaire (IPQ-R). Psychology \& Health, 17(1), 1-16. https://doi.org/10.1080/08870440290001494

Mukhtar, S. (2020). Psychological health during the coronavirus disease 2019 pandemic outbreak. International Journal of Social Psychiatry, 66(5), 1-5. https://doi.org/10.1177/0020764020925835

Pérez-Bautista, Y. Y., \& Lugo-González, I. V. (2017). Aplicación de un instrumento de conducta sexual a través de dos métodos de recolección de datos. Trabajo presentado en el Sexto Seminario Internacional sobre Comportamiento y Aplicaciones (SINCA), Tlaxcala, México.

Pérez, E., Medrano, L. A., \& Rosas, J. S. (2013). El path analysis: conceptos básicos y ejemplos de aplicación. Revista Argentina de Ciencias del Comportamiento, 5(1), 52-66. https://doi.org/10.32348/1852.4206.v5.n 1.5160

Pérez-Fuentes, M. D. C., Molero-Jurado, M. D. M., Oropesa-Ruiz, N. F., Martos-Martínez, Á., Simón Márquez, M. D. M., Herrera-Peco, I., \& Gázquez Linares, J. J. (2020). Questionnaire on Perception of Threat from COVID-19. Journal of Clinical Medicine, 9(4), 1196-1207. https://doi.o $\mathrm{rg} / 10.3390 / \mathrm{jcm} 9041196$

Pérez-Gay Juárez, F., Reynoso-Alcántara, V., Flores González. R., ContrerasIbáñez, C., Castro-López, C., Martínez, L., \& The COVID-Stress International Collaboration (2020). Evaluación del Estrés frente a la Pandemia del COVID-19 en población mexicana. Reporte de los resultados de la encuesta global COVIDISTRESS. https://www.uv.mx/c entrodeopinion/files/2020/05/Reporte-Descriptivo-para-OSF2.pdf

Ramos-Lira, L., Rafful, Claudia., Flores-Celis, K., Mora-Ríos, J., GarcíaAndrade, C., Rascón-Gasca, M. L., Bautista-Aguilar, N., \& CervantesMuñoz, C. (2020). Emotional responses and coping strategies in adult Mexican population during the first lockdown of the COVID-19 pandemic: An exploratory study by sex. Salud Mental, 43(6), 243-251. ht tps://doi.org/10.17711/SM.0185-3325.2020.034 
Raude, J., Lecrique, J. M., Lasbeur, L., Leon, C., Guignard, R., Roscoät, E. D., \& Arwidson, P. (2020). Determinants of preventive behaviors in response to the COVID-19 pandemic in France: Comparing the sociocultural, psychosocial and social cognitive explanations. Frontiers in Psychology, 11, 3345. https://doi.org/10.3389/fpsyg.2020.584500

Rosenstock, I. M. (1974). Historical origins of the health belief model. Health Education Monographs, 2(4), 328-335. https://doi.org/10.1177/109019 817400200403

Rubin, G. J., Potts, H. W. W., \& Michie S. (2010). The impact of communications about swine flu (influenza A H1N1v) on public responses to the outbreak: Results from 36 national telephone surveys in the UK. Health Technology Assessment, 14(34):183-266. https://doi.org /10.3310/hta14340-03

Samaniego, A., Urzúa, A., Buenahora, M., \& Vera-Villarroel, P. (2020). Sintomatología asociada a trastornos de salud mental en trabajadores sanitarios en Paraguay: efecto COVID-19. Revista Interamericana de Psicología, 54(1), e1298. https://doi.org/10.30849/ripijp.v54i1.1298

Sandín, B., Valiente, R. M., García-Escalera, J., \& Chorot, P. (2020). Impacto psicológico de la pandemia de COVID-19: efectos negativos y positivos en población española asociados al periodo de confinamiento nacional. Revista Psicopatología y Psicología Clínica, 1(1), 1-22. https://doi.org/10. 5944/rppc.27569

Secretaría de Salud (SSa, 2020). Todo sobre el COVID-19. Disponible en https ://coronavirus.gob.mx/

Shahnazi, H., Ahmadi-Livani, M., Pahlavanzadeh, B., Rajabi, A., Hamrah, M. S., \& Charkazi, A. (2020). Assessing preventive health behaviors from COVID-19: A cross sectional study with health belief model in Golestan Province, Northern of Iran. Infectious Diseases of Poverty, 9(1), 1-9. https ://doi.org/10.1186/s40249-020-00776-2

Stirratt, M. J., Dunbar-Jacob, J., Crane, H. M., Simoni, J. M., Czajkowski, S., Hilliard, M. E., Aikens, J. E., Hunter, C. M., Velligan, D. I., Huntley, K., Ogedegbe, G., Rand, C., Schron, E., \& Nilsen, W. J. (2015). Selfreport measures of medication adherence behavior: Recommendations on optimal use. Translational Behavioral Medicine, 5(4), 470-482. https://d oi.org/10.1007/s13142-015-0315-2

Taylor, S. (2019). The psychology of pandemics: Preparing for the next global outbreak of infectious disease. Cambridge Scholars Publishing.

Teslya, A., Pham, T. M., Godijk, N. G., Kretzschmar, M. E., Bootsma, M. C., \& Rozhnova, G. (2020). Impact of self-imposed prevention measures and short-term government-imposed social distancing on mitigating and delaying a COVID-19 epidemic: A modelling study. PLoS medicine, 17(7), e1003166. https://doi.org/10.1371/journal.pmed.1003166

Unni, E., \& Shiyanbola, O. O. (2016). Clustering medication adherence behavior based on beliefs in medicines and illness perceptions in patients taking asthma maintenance medications. Current Medical Research and Opinion, 32(1), 113-121. https://doi.org/10.1185/03007995.2015.1105 204

Urzúa, A., Samaniego, A., Caqueo-Urízar, A., Zapata, A., \& Irarrazaval, M. (2020b). Problemas de salud mental en trabajadores de la salud durante la 
pandemia por COVID-19. Revista Médica de Chile, 148, 1121-1127. htt ps://dx.doi.org/10.4067/S0034-98872020000801121

Urzúa, A., Vera-Villarroel, P., Caqueo-Urízar, A., \& Polanco, R. (2020a). La psicología en la prevención y manejo del COVID-19. Aportes desde la evidencia inicial. Terapia Psicológica, 38(1), 103-118. http://dx.doi.org/1 0.4067/S0718-48082020000100103

Wang, C., Pan, R., Wan, X., Tan, Y., Xu, L., Ho, C. S., \& Ho, R. C. (2020). Immediate psychological responses and associated factors during the initial stage of the 2019 coronavirus disease (COVID-19) epidemic among the general population in China. International Journal of Environmental Research and Public Health, 17(5), 1729. https://doi.org/ 10.3390/ijerph17051729

Weigold, A., Weigold, I. K., \& Russell, E. J. (2013). Examination of the equivalence of self-report survey-based paper-and-pencil and internet data collection methods. Psychological Methods, 18(1), 53-70. https://doi.org/ $10.1037 / \mathrm{a} 0031607$

West, R., Michie, S., Rubin, G. J., \& Amlôt, R. (2020). Applying principles of behaviour change to reduce SARS-CoV-2 transmission. Nature Human Behaviour, 1-9. https://doi.org/10.1038/s41562-020-0887-9

Yang, S., \& Cho, S. I. (2017). Middle East respiratory syndrome risk perception among students at a university in South Korea, 2015. American Journal of Infection Control, 45(6), e53-e60. http://dx.doi.org/10.1016/j.ajic.2017. 02.013 